\title{
Jet-induced star formation: Good news from big, bad black holes
}

\author{
Wil van Breugel ${ }^{1}$, Chris Fragile ${ }^{1}$, Stephen Croft $^{1}$ \\ Wim de Vries $^{1,2}$, Peter Anninos ${ }^{1}$ and Stephen Murray ${ }^{1}$ \\ ${ }^{1}$ University of California, Lawrence Livermore National Laboratory, L-413, P.O. Box 808, \\ Livermore, CA 94550,USA \\ ${ }^{2}$ University of California at Davis, One Shields Ave, Davis, CA 95616, USA \\ email: wil@igpp.ucllnl.org,fragile1@llnl.gov,scroft@igpp.ucllnl.org, \\ wdevries@igpp.ucllnl.org,anninos1@llnl.gov,murray8@llnl.gov
}

\begin{abstract}
We discuss obbservations and numerical simulations which show that radiative shocks in jet-cloud collisions can trigger the collapse of intergalactic clouds and subsequent star formation in low luminosity, 'FR-I' type, radio galaxies.
\end{abstract}

\section{Introduction}

Many, if not all, galaxies have massive - supermassive $\left(10^{6}-10^{9}\right.$ Solar mass $)$ black holes at their centers. These black holes become 'active' sources of energy - Active Galactic Nuclei (AGN) - when they accrete material from their surroundings in hot accretion disks. These accretion disks emit high energy radiation and particles, drive high velocity winds and can form collimated, relativistic, jets. Heating, radiative ablation, and shocks will affect the ambient interstellar medium (ISM) or even intergalactic medium (IGM) out to very large distances. High pressure starbursts (100 - 1000 times our Galaxy) produce large numbers of supernovae which affect their surrounding ISM in similar ways.

This high energy feedback from active black holes and exploding stars may have a profoud effect on the ISM in their parent galaxies. In fact, it has been proposed (Silk and Rees 1998) that this might self-regulate galaxy and black hole growth and explain the observed close correlation between the masses of supermassive black holes and the stellar bulges of their parent galaxies (e.g. Magorrian et al. 1998). Studies of high energy feedback on interstellar and intergalactic media are therefore an important part of observational and theoretical work on the formation and evolution of galaxies and their central black holes.

Most of this work focuses on 'negative' feedback, where intense radiation fields and starburst winds limit furher star formation and galaxy/black hole growth. But AGN may also provide 'positive' feedback. In particular, jets and lobes from radio sources may drive radiative shocks into dense clouds triggering extra star formation.

Radio jets can trigger star formation in two different ways. Although the powerful, collimated jets in 'FR-II' type (Fanaroff and Riley 1974) radio galaxies punch through clouds in the ISM/IGM, their slowly expanding radio lobes can trigger star formation in cocoons around them (Bicknell et al. 2000). On the other hand the less powerful jets in 'FR-I' type radio galaxies may trigger star formation even in head-on cloud collisions (Fig. 1; van Breugel et al. 1985; Croft et al. 2004). In general, in the early Universe, when galaxies were still forming and gas densities were much higher, jet-induced star formation may have been relatively common. Evidence for that is strongly suggested by the 


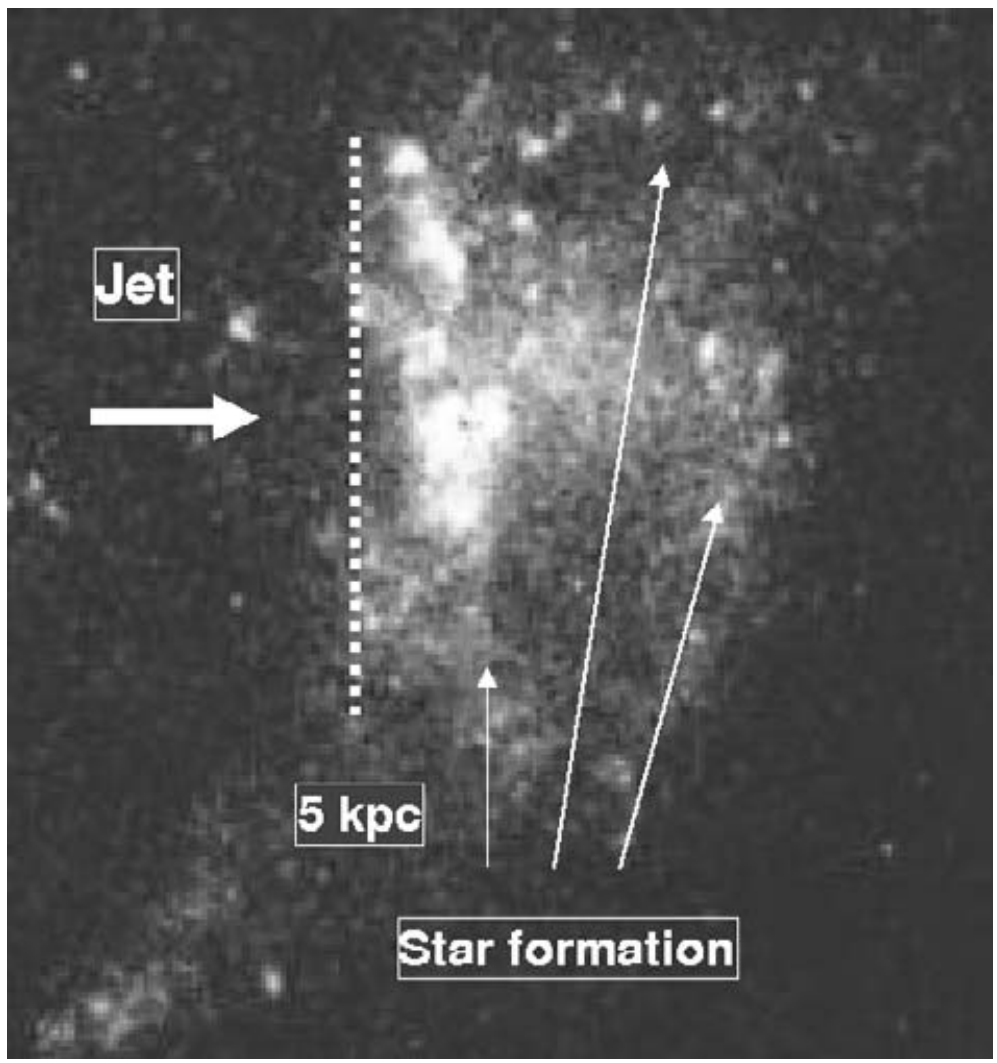

Figure 1. HST observation of induced star formation at end of a jet (Croft et al. 2004)

discovery of the radio/optical alignment effect in very distant radio galaxies (McCarthy et al. 1987; Chambers et al 1987).

\section{Jet-induced star formation in nearby radio galaxies}

The first evidence for jet-induced star formation radio galaxies was found in the nearest, FR-I type, radio galaxy Centaurus A. Recent observations with the Hubble Space Telescope have confirmed that there are about half a dozen young $(<15 \mathrm{Myr}) \mathrm{OB}$ associations near filaments of ionized gas located between the radio jet and a large HI cloud (Mould et al. 2000). Star forming regions associated with radio sources have also been found in cooling flow clusters, with the best example being in Abell 1795 (McNamara 2002).

One of the most spectacular observed jet-induced starbursts is 'Minkowski's Object' associated with the elliptical galaxy NGC 541 in the cluster of galaxies Abell 194 (Fig. 1). Its morphology is strongly suggestive of a collision between the FR-I type jet from NGC 541 and a dense cloud: M.O. has the same overall diameter as the jet, appears wrapped around the end of the jet, has bright emission in the upstream direction and filamentary structure down-stream where the jet appears disrupted. Spectroscopically M.O. looks like an HII region, resembling starburst galaxies. The $\mathrm{H} \alpha$ luminosity suggests a modest star formation rate of $0.3 \mathrm{M}_{\odot} / \mathrm{yr}$. VLA observations (van Breugel and van Gorkom, unpublished) show two detections down-stream from the jet-cloud collision site, indicating a total $\mathrm{HI}$ mass of $\sim 3 \times 10^{8} \mathrm{M}_{\odot}$. 


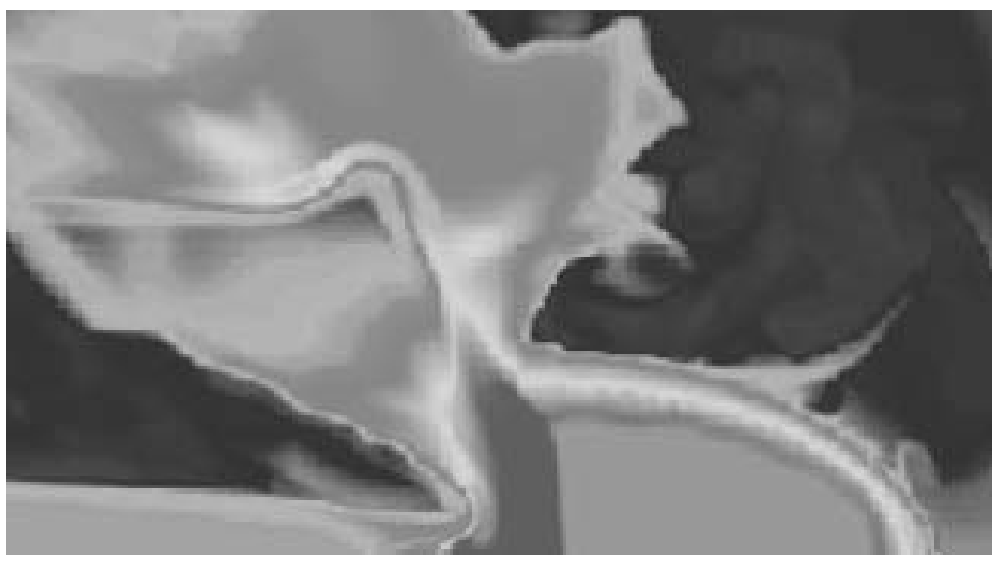

Figure 2. Numerical simulation of jet / cloud collision using COSMOS (Fragile et al 2004). Radiative shocks trigger star formation at front (left) and the sides

\section{Numerical simulations of jet-cloud collisions}

Numerical simulations are essential if we want to learn more about the physical conditions in radio jets and their environment (density, temperature, composition etc.). For example, we don't even know what is inside these jets $\left(\mathrm{e}^{-} \mathrm{e}^{+}\right.$?; $\mathrm{e}^{-} \mathrm{p}^{+}$?; Hujeirat 2004), and direct knowledge about the physical conditions in forming galaxies will always be limited because of their faintness. Previous numerical simulations by several other groups did not include effects of radiative cooling, magnetic fields etc. Radiative cooling is important because it allows compressed clouds to collapse and break up in numerous, dense cold fragments which survive for many timescales and are presumably the precursors to star formation (Fragile et al 2004; Mellema et al 2002). Previous work has also focused primarily on FR-II type radio galaxies even though, because of their proximity, much better observational data can be obtained for FR-I type jet-induced starbursts such as M.O.

Fragile et al (2004) used the LLNL developed, multi-dimensional, multi-physics, massively parallel 'COSMOS' numerical simulations package to investigate both FR-I and FR-II type jet-induced star formation systems. To simulate the jet induced star formation in M.O. Fragile et al (2004) assumed that NGC 541 is surrounded by a multi-phase medium resembling cluster atmospheres (e.g. Ferland et al. 2002). Specifically, it was assumed that the FR-I jet interacts with a moderate density, hot 'mother'-cloud with a semimajor axis of $10 \mathrm{kpc}$, a semiminor axis of $5 \mathrm{kpc}$, a density $n_{m c l}=0.1 \mathrm{~cm}^{-3}$, and temperature $T=10^{6} \mathrm{~K}$. This corresponds to an initial total cloud mass of $\approx 10^{9} M_{\odot}$. Within this mother-cloud denser, warm clouds were assumed to be embedded with typical sizes of $100 \mathrm{pc}, n_{c l}=10 \mathrm{~cm}^{-3}$, and temperature $T=10^{4} \mathrm{~K}$. The detailed radio and X-ray study of the proto-typical FRI-type radio galaxy 3C31 by Laing and Bridle (2002) was used to estimate plausible jet parameters near M.O., at $\sim 15 \mathrm{kpc}$ from the NGC 541 AGN.

The collision with the mother-cloud triggers a nearly planar shock down the long axis of the cloud (Fig. 2). As the bow shock from the jet wraps around, it also triggers shocks along the sides of the cloud. This may explain the filamentary nature of the star-forming region seen in M.O. downstream from the jet (Fig. 1). To determine whether the clouds embedded within the mother-cloud would indeed collapse and form stars we ran a number of simulations to explore the density / velocity parameter space. We found that for shock 
velocities between $v_{s h}=1,000-10,000 \mathrm{~km} \mathrm{~s}^{-1}$ and $n_{c l}=1-100 \mathrm{~cm}^{-3}$ the cooling times were sufficiently short that star formation could occur.

\section{Conclusions}

Our results show that M.O. could be due to star formation which was triggered by the collapse of dense Intergalactic Medium (IGM) gas clouds due to a collision with the jet. Star formation in the IGM near expanding radio lobes has been seen in 'cooling' flow clusters (McNamara 2002). Although A 194 is a not a cooling flow cluster, extended X-ray emission has been detected with ROSAT and extends along a prominent stellar bridge which connects three large ellipticals: NGC 541 and its double companion galaxy NGC 545/547 associated with 3C40 to the North-East (Lazzati et al. 1998; Nikogossyan et al. 1999). M.O. appears to be located in this bridge, where regions of overdense gas would not be unexpected. Further observations to search for evidence of overdense gas in this stellar bridge are underway.

\section{Acknowledgements}

This work was performed under the auspices of the U.S. Department of Energy, National Nuclear Security Administration by the University of California, Lawrence Livermore National Laboratory under contract No. W-7405-Eng-48. W.v.B. also acknowledges NASA grants GO 9779 and GO3-4150X in support of high-redshift radio galaxy research with HST and Chandra.

\section{References}

Bicknell, G., et al. 2000, ApJ, 540, 678

Chambers, K., et al. 1987, Nature, 329, 604

Croft, S., et al. 2004, in prep

Fanaroff, B. L., \& Riley, J. M. 1974, MNRAS, 167, 31P

Ferland, G. J., et al. 2002, MNRAS, 333, 876

Fragile, P. C., et al. 2004, ApJ, 604, 74

Hujeirat, A. 2004, A\&A, 416, 423

Laing, R. A., \& Bridle, A. H. 2002, MNRAS, 336, 1161

Lazzati, D., et al. 1998, A\&A, 339, 52

Mould, J. R., et al. 2000, ApJ, 536, 266

Magorrian, J. 1998, AJ, 115, 2285

McCarthy, P. J., et al. 1987, ApJ, 321, L29

McNamara, B. R. 2002, New Astronomy Review, 46, 141

Mellema, G., et al. 2002, A\&A, 395, L13

Nicogossyan, E., et al. 1999, A\&A, 349, 97

Silk, J. \& Rees, M. 1998, A\&A, 331, L1

van Breugel, W., et al. 1985, ApJ, 293, 83 